\title{
Internal-Model-Principle-Based Specific Harmonics Repetitive Controller for Grid-Connected PWM Inverters
}

\author{
Wenzhou Lu, ${ }^{1}$ Jinfei Shen, ${ }^{1}$ and Yunhu Yang ${ }^{2}$ \\ ${ }^{1}$ Key Laboratory of Advance Process Control for Light Industry, Ministry of Education, Jiangnan University, Wuxi 214122, China \\ ${ }^{2}$ School of Electrical and Information Engineering, Anhui University of Technology, Maianshan 243002, China
}

Correspondence should be addressed to Wenzhou Lu; luwenzhou@126.com

Received 9 May 2016; Revised 22 July 2016; Accepted 9 August 2016

Academic Editor: Alfonso Banos

Copyright (C) 2016 Wenzhou Lu et al. This is an open access article distributed under the Creative Commons Attribution License, which permits unrestricted use, distribution, and reproduction in any medium, provided the original work is properly cited.

\begin{abstract}
This paper analyzes the general properties of IMP-based controller and presents an internal-model-principle-based (IMP-based) specific harmonics repetitive control (SHRC) scheme. The proposed SHRC is effective for specific $n k \pm m$ order harmonics, with $n>m \geq 0$ and $k=0,1,2, \ldots$ Using the properties of exponential function, SHRC can also be rewritten into the format of multiple resonant controllers in parallel, where the control gain of SHRC is $n / 2$ multiple of that of conventional RC (CRC). Therefore, including SHRC in a stable closed-loop feedback control system, asymptotic disturbance eliminating, or reference tracking for any periodic signal only including these specific harmonic components at $n / 2$ times faster error convergence rate compared with CRC can be achieved. Application examples of SHRC controlled three-phase/single-phase grid-connected PWM inverters demonstrate the effectiveness and advantages of the proposed SHRC scheme.
\end{abstract}

\section{Introduction}

Repetitive control (RC), as an internal model principle (IMP) $[1,2]$ based controller, can track or eliminate periodic signals in an effective way if it is included in a stable closed-loop system. Conventional RC (CRC) [3], presented since the early 1980 and being the most widely used RC format nowadays, can achieve zero-error tracking or disturbance rejection for any periodic signal whose fundamental period time is known at first. Through fast-Fourier transformation (FFT) analysis, any known periodic signal can be decomposed into dc, fundamental component, and all harmonic components. That is, CRC is effective to track or eliminate any periodic signal including any order harmonic. Due to the time delay line being included in the RC structure, CRC is slower than any instantaneous feedback controller although it has better tracking accuracy. RC has been studied in several aspects [4-12], including its properties and structures. Moreover, its applications can be found in many fields, such as disc drives [13], robots [14], satellites [15], and PWM converters [8, 9, 1622].

However, in many applications $[7,8,10]$, dominant harmonics only concentrate at some specific order harmonic frequencies. Especially, in three-phase/single-phase PWM inverter applications, $6 k \pm 1$ or $4 k \pm 1(k=1,2, \ldots)$ order harmonics dominate their output voltages/currents distortions. In these cases, CRC might be too slow to track/eliminate these specific harmonics with satisfactory error convergence rate.

In order to solve this problem, this paper proposes an IMP-based specific harmonics RC (SHRC) for threephase/single-phase grid-connected PWM inverters. To better understand the proposed SHRC, the general properties of IMP-based controller and the performance of IMP-based CRC are analyzed at first. Then, the performance of IMPbased SHRC and its error convergence rate analysis are also given. Finally, two application cases of SHRC controlled three-phase/single-phase grid-connected PWM inverters are provided to demonstrate the effectiveness and advantages of the proposed SHRC scheme.

\section{IMP-Based Controller}

2.1. Internal Model Principle (IMP). Some specific disturbance and reference signals can be described as the output of a linear dynamic system with zero-input and certain 


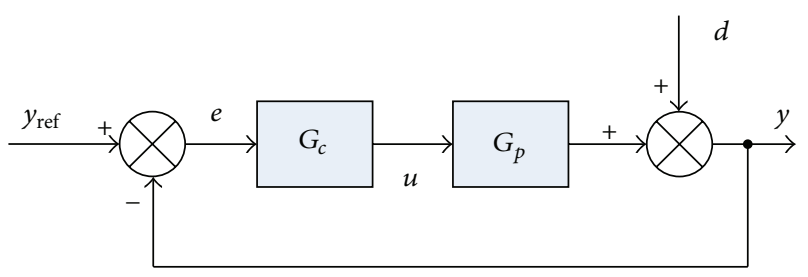

FIgURE 1: Closed-loop feedback control system.

initial conditions. Thus, a general disturbance signal can be described as the format of a differential equation as follows:

$$
\frac{d^{q} d(t)}{d t^{q}}+\sum_{i=0}^{q-1} \gamma_{i} \frac{d^{i} d(t)}{d t^{i}}=0
$$

Using Laplace transform, (1) can be derived into

$$
d(s)=\frac{N_{d}(s) x_{d}(0)}{\Gamma_{d}(s)}
$$

where $\Gamma_{d}(s)$ is the disturbance generating polynomial (D-GP), which can be defined into

$$
\Gamma_{d}(s) \triangleq s^{q}+\sum_{i=0}^{q-1} \gamma_{i} s^{i}
$$

Notice that this D-GP is only related with the denominator of (2). Similarly, reference signal can be described in the same way, as long as replacing the symbol " $d$ " with " $r$." Reference generating polynomial (R-GP) $\Gamma_{r}(s)$ can be defined as follows:

$$
\Gamma_{r}(s) \triangleq s^{q}+\sum_{i=0}^{q-1} \lambda_{i} s^{i}
$$

Then, internal model principle (IMP) $[2,23]$ can be expressed as follows: If disturbance $d(t)$ or reference input $y_{\text {ref }}(t)$ can be expressed as its D-GP or R-GP just like $\Gamma_{d}(s)$ or $\Gamma_{r}(s)$, asymptotic error elimination or reference tracking can be achieved for the control system using the following controller:

$$
G_{c}(s)=\frac{P(s)}{\Gamma_{d}(s) \bar{L}(s)},
$$

or

$$
G_{c}(s)=\frac{P(s)}{\Gamma_{r}(s) \bar{L}(s)} .
$$

Therefore, only D-GP or R-GP, that is, $\Gamma_{d}(s)$ or $\Gamma_{r}(s)$, is needed when using IMP, while the amplitude information of disturbance or reference input is not needed.

2.2. The General Properties of IMP-Based Controller. Figure 1 shows the diagram of a closed-loop feedback control system. Let the model of plant be

$$
G_{p}(s)=\frac{B_{o}(s)}{A_{o}(s)}=\frac{b_{n-1} s^{n-1}+b_{n-2} s^{n-2}+\cdots+b_{0}}{s^{n}+a_{n-1} s^{n-1}+a_{n-2} s^{n-2}+\cdots+a_{0}}
$$

and assume that $\Gamma_{d}(s)$ or $\Gamma_{r}(s)$ is not the factor of $B_{o}(s)$.
Then, the output sensitivity function of closed-loop feedback system shown in Figure 1 is

$$
S_{o}(s)=\frac{\Gamma_{d}(s) \bar{L}(s) A_{o}(s)}{\Gamma_{d}(s) \bar{L}(s) A_{o}(s)+P(s) B_{o}(s)}
$$

or

$$
S_{o}(s)=\frac{\Gamma_{r}(s) \bar{L}(s) A_{o}(s)}{\Gamma_{r}(s) \bar{L}(s) A_{o}(s)+P(s) B_{o}(s)}
$$

and the output complementary sensitivity function is

$$
T_{o}(s)=1-S_{o}(s)=\frac{P(s) B_{o}(s)}{\Gamma_{d}(s) \bar{L}(s) A_{o}(s)+P(s) B_{o}(s)}
$$

or

$$
T_{o}(s)=1-S_{o}(s)=\frac{P(s) B_{o}(s)}{\Gamma_{r}(s) \bar{L}(s) A_{o}(s)+P(s) B_{o}(s)} .
$$

Assume the selected $\bar{L}(s)$ and $P(s)$ can make the closedloop characteristic equation

$$
A_{\mathrm{cl}}(s)=\Gamma_{d}(s) \bar{L}(s) A_{o}(s)+P(s) B_{o}(s)
$$

or

$$
A_{\mathrm{cl}}(s)=\Gamma_{r}(s) \bar{L}(s) A_{o}(s)+P(s) B_{o}(s)
$$

have the negative realistic roots. Thus, the output response of disturbance $d(t)$ can be obtained as

$$
y(s)=S_{o}(s) d(s)=\frac{\bar{L}(s) A_{o}(s) N_{d}(s) x_{d}(0)}{A_{\mathrm{cl}}(s)} .
$$

Because $A_{\mathrm{cl}}(s)$ has stable roots, the inverse Laplace transform of $y(s)$ can asymptotically converge to zero; that is, $y(t \rightarrow \infty)=0$.

The system error response of disturbance $d(t)$ is

$$
e(s)=-S_{o}(s) d(s)=-\frac{\bar{L}(s) A_{o}(s) N_{d}(s) x_{d}(0)}{A_{\mathrm{cl}}(s)} .
$$

Because $A_{\mathrm{cl}}(s)$ has stable roots, the inverse Laplace transform of $e(s)$ can asymptotically converge to zero; that is, $e(t \rightarrow \infty)=0$.

The system error response of reference $y_{\text {ref }}(t)$ is

$$
e(s)=S_{o}(s) y_{\mathrm{ref}}(s)=\frac{\bar{L}(s) A_{o}(s) N_{r}(s) x_{r}(0)}{A_{\mathrm{cl}}(s)} .
$$

Because $A_{\mathrm{cl}}(s)$ has stable roots, the inverse Laplace transform of $e(s)$ can asymptotically converge to zero; that is, $e(t \rightarrow \infty)=0$.

The system output response of reference $y_{\text {ref }}(t)$ is

$$
\begin{aligned}
y(s) & =T_{o}(s) y_{\text {ref }}(s) \\
& =y_{\text {ref }}(s)-\frac{\bar{L}(s) A_{o}(s) N_{r}(s) x_{r}(0)}{A_{\mathrm{cl}}(s)} .
\end{aligned}
$$




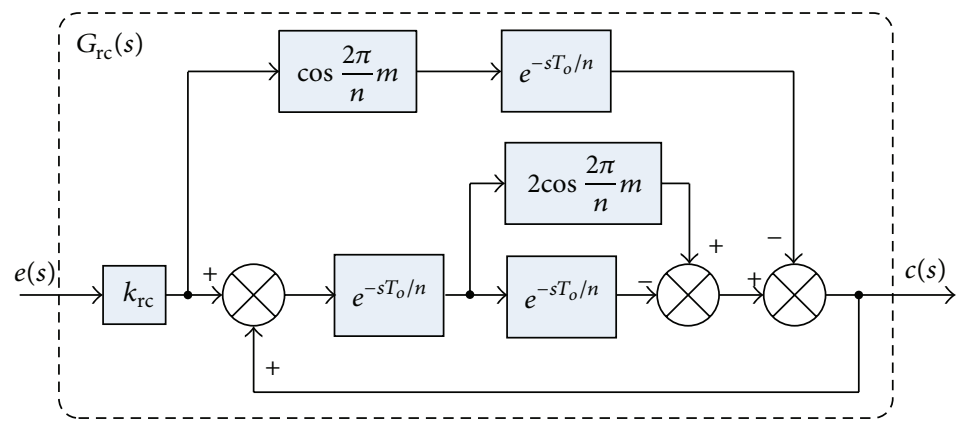

FIGURE 2: Specific harmonics repetitive control (SHRC).

Because $A_{\mathrm{cl}}(s)$ has stable roots, the inverse Laplace transform of $y(s)$ can asymptotically track its reference input; that is, $y(t) \rightarrow y_{\text {ref }}(t)$.

Therefore, if D-GP or R-GP is included in the denominator of a controller, asymptotic disturbance eliminating or reference tracking can be achieved for the control system.

Then, we will see why conventional RC (CRC) is an IMPbased controller and why it can track/eliminate all harmonics.

2.3. IMP-Based Conventional Repetitive Controller (CRC). According to the properties of exponential function [24], CRC can be rewritten into

$$
\begin{aligned}
G_{\mathrm{rc}}(s) & =k_{\mathrm{rc}} \cdot \frac{e^{-s T_{o}}}{1-e^{-s T_{o}}}=k_{\mathrm{rc}} \cdot \frac{e^{-s T_{o} / 2}}{2 \sinh \left(s T_{o} / 2\right)} \\
& =k_{\mathrm{rc}} \cdot \frac{e^{-s T_{o} / 2}}{s T_{o} \cdot \prod_{k=1}^{\infty}\left[1+s^{2} /\left(k^{2} \omega_{o}^{2}\right)\right]},
\end{aligned}
$$

where $k_{\mathrm{rc}}$ is the control gain and $T_{o}=2 \pi / \omega_{o}=1 / f_{o}$ is the fundamental period of signals with $f_{o}$ being the fundamental frequency, $\omega_{o}$ being the fundamental angular frequency.

If the disturbance $d(t)$ or reference input $y_{\text {ref }}(t)$ of closedloop feedback control system is a periodic signal, which can be decomposed into the summation of all harmonic components, that is, $\mathrm{dc}$, fundamental component and any order harmonic component using FFT, its D-GP or R-GP, that is, $\Gamma_{d}(s)$ or $\Gamma_{r}(s)$, can be derived as follows:

$$
\begin{aligned}
d(t) & =d_{0}+\sum_{k=1}^{\infty} d_{k} \cos \left(k \omega_{o} t+\varphi_{k}\right) \Longrightarrow \\
\Gamma_{d}(s) & =s \prod_{k=1}^{\infty}\left(s^{2}+\left(k \omega_{o}\right)^{2}\right)
\end{aligned}
$$

or

$$
\begin{aligned}
y_{\text {ref }}(t) & =r_{0}+\sum_{k=1}^{\infty} r_{k} \cos \left(k \omega_{o} t+\varphi_{k}^{\prime}\right) \Longrightarrow \\
\Gamma_{r}(s) & =s \prod_{k=1}^{\infty}\left(s^{2}+\left(k \omega_{o}\right)^{2}\right) .
\end{aligned}
$$

Then, CRC can be derived into

$$
G_{c}(s)=G_{\mathrm{rc}}(s)=\frac{k_{\mathrm{rc}} e^{-s T_{o} / 2} \prod_{k=1}^{\infty}\left(k^{2} \omega_{o}^{2}\right)}{T_{o} \cdot \Gamma_{d}(s)}
$$

or

$$
G_{c}(s)=G_{\mathrm{rc}}(s)=\frac{k_{\mathrm{rc}} e^{-s T_{o} / 2} \prod_{k=1}^{\infty}\left(k^{2} \omega_{o}^{2}\right)}{T_{o} \cdot \Gamma_{r}(s)} .
$$

From (21) or (22), it can be seen that D-GP $\Gamma_{d}(s)$ or R-GP $\Gamma_{r}(s)$ is included in the denominator of CRC controller. Thus, asymptotic disturbance eliminating or reference tracking for any periodic signal can be achieved using IMP indicated in Section 2.1, if CRC is used as the controller in a closedloop feedback control system. Therefore, CRC is an IMPbased controller and is effective for any periodic signal that is composed with all harmonic components.

\section{SHRC}

3.1. Specific Harmonics RC (SHRC). A specific harmonics RC (SHRC) shown in Figure 2 for efficient removing/tracking specific $n k \pm m$ order harmonics is proposed as follows:

$$
G_{\mathrm{rc}}(s)=k_{\mathrm{rc}} \cdot \frac{\cos (2 \pi m / n) e^{s T_{o} / n}-1}{e^{2 s T_{o} / n}-2 \cos (2 \pi m / n) e^{s T_{o} / n}+1},
$$

where $n$ and $m$ are integers with $n>m \geq 0$.

In three-phase/single-phase PWM inverter applications, because $6 k \pm 1$ or $4 k \pm 1(k=1,2, \ldots)$ order harmonics dominate its output distortion, (23) can be used for these specific harmonics with $n=6$ or $n=4$ and $m=1$.

Using the similar analysis with CRC in Section 2.3, it can be achieved that SHRC is also an IMP-based controller in the following subsection.

3.2. IMP-Based SHRC. According to the properties of exponential function [24], (23) can be rewritten into 


$$
\begin{aligned}
G_{\mathrm{rc}}(s) & =\frac{k_{\mathrm{rc}}}{2} \cdot \frac{\cos (2 \pi m / n)-e^{-s T_{o} / n}}{\cosh \left(s T_{o} / n\right)-\cos (2 \pi m / n)} \\
& =\frac{k_{\mathrm{rc}} \cdot\left[\cos ((2 \pi / n) m)-e^{-s\left(T_{o} / n\right)}\right]}{\left\{4 \sin ^{2}(m \pi / n)\left(1+s^{2} /\left(m^{2} \omega_{o}^{2}\right)\right) \cdot \prod_{k=1}^{\infty}\left\{\left[1+s^{2} /\left((n k+m)^{2} \omega_{o}^{2}\right)\right]\left[1+s^{2} /\left((n k-m)^{2} \omega_{o}^{2}\right)\right]\right\}\right\}},
\end{aligned}
$$

where $m \neq 0$. If $m=0,(23)$ can be rewritten into

$$
\begin{aligned}
G_{\mathrm{rc}}(s) & =\frac{k_{\mathrm{rc}}}{e^{s T_{o} / n}-1} \\
& =\frac{k_{\mathrm{rc}}}{\left(s T_{o} / n\right) \cdot e^{s T_{o} / 2 n} \cdot \prod_{k=1}^{\infty}\left[1+s^{2} /\left(n k \omega_{0}\right)^{2}\right]} .
\end{aligned}
$$

If the disturbance signal $d(t)$ of closed-loop feedback control system is a periodic signal only including specific $n k \pm m$ order harmonic components, its D-GP, that is, $\Gamma_{d}(s)$, can be derived as follows:

$$
\begin{aligned}
& d(t)= \begin{cases}d_{0}+\sum_{k=1}^{\infty} d_{n k} \cos \left(n k \omega_{o} t+\varphi_{n k}\right) & (m=0) \\
\sum_{k=1}^{\infty} d_{n k-m} \cos \left((n k-m) \omega_{o} t+\varphi_{n k-m}\right)+\sum_{k=0}^{\infty} d_{n k+m} \cos \left((n k+m) \omega_{o} t+\varphi_{n k+m}\right) & (m \neq 0) .\end{cases} \\
& \Gamma_{d}(s)= \begin{cases}s \prod_{k=1}^{\infty}\left(s^{2}+\left(n k \omega_{o}\right)^{2}\right) & (m=0) \\
\left(s^{2}+\left(m \omega_{o}\right)^{2}\right) \prod_{k=1}^{\infty}\left\{\left(s^{2}+\left((n k-m) \omega_{o}\right)^{2}\right) \cdot\left(s^{2}+\left((n k+m) \omega_{o}\right)^{2}\right)\right\} & (m \neq 0) .\end{cases}
\end{aligned}
$$

Then, SHRC can be derived into

$$
G_{c}(s)=G_{\mathrm{rc}}(s)= \begin{cases}k_{\mathrm{rc}} \cdot \frac{n \cdot e^{-s T_{o} / 2 / n} \prod_{k=1}^{\infty}\left(n k \omega_{o}\right)^{2}}{T_{o} \cdot \Gamma_{d}(s)} & (m=0) \\ \frac{k_{\mathrm{rc}}\left[\cos (2 \pi m / n)-e^{-s T_{o} / n}\right] m^{2} \omega_{o}^{2} \prod_{k=1}^{\infty}\left[(n k+m)^{2}(n k-m)^{2} \omega_{o}^{4}\right]}{4 \sin ^{2}(m \pi / n) \cdot \Gamma_{d}(s)} & (m \neq 0) .\end{cases}
$$

Similarly, if the reference input $y_{\text {ref }}(t)$ is a periodic signal only including specific $n k \pm m$ order harmonic components, its R-GP, that is, $\Gamma_{r}(s)$, and corresponding SHRC can also be derived.

So, it can be seen that D-GP $\Gamma_{d}(s)$ or R-GP $\Gamma_{r}(s)$ is included in the denominator of SHRC controller. Thus, asymptotic disturbance eliminating or reference tracking for any periodic signal only including these specific $n k \pm m$ order harmonic components can be achieved using IMP indicated in Section 2.1, if SHRC is used as the controller in a closedloop feedback control system. Therefore, SHRC is also an IMP-based controller and is effective for any periodic signal only including these specific harmonics.

3.3. Error Convergence Rate of SHRC. According to the properties of exponential function [24], SHRC can also be rewritten into the format of multiple resonant controllers in parallel as follows:

$$
\begin{aligned}
& G_{\mathrm{rc}}(s)=\frac{1}{2} k_{\mathrm{rc}} \cdot\left[\frac{e^{j(2 \pi / n) m}}{e^{s\left(T_{o} / n\right)}-e^{j(2 \pi / n) m}}\right. \\
& \left.+\frac{e^{-j(2 \pi / n) m}}{e^{s\left(T_{o} / n\right)}-e^{-j(2 \pi / n) m}}\right]=k_{\mathrm{rc}} \cdot\left\{-\frac{1}{2}\right. \\
& +\frac{n}{2 T_{o}\left(s-j m \omega_{o}\right)}+\frac{n}{2 T_{o}\left(s+j m \omega_{o}\right)} \\
& +\frac{n}{2 T_{o}} \sum_{k=1}^{+\infty} \frac{2\left(s-j m \omega_{o}\right)}{\left(s-j m \omega_{o}\right)^{2}+n^{2} k^{2} \omega_{o}^{2}} \\
& \left.+\frac{n}{2 T_{o}} \sum_{k=1}^{+\infty} \frac{2\left(s+j m \omega_{o}\right)}{\left(s+j m \omega_{o}\right)^{2}+n^{2} k^{2} \omega_{o}^{2}}\right\} .
\end{aligned}
$$

From (28), it can be achieved that, for specific $n k \pm m$ order harmonics, the control gain of SHRC is $(n / 2) k_{\mathrm{rc}} / T_{o}$. From [25], the corresponding control gain of CRC is $k_{\mathrm{rc}} / T_{o}$. So, 


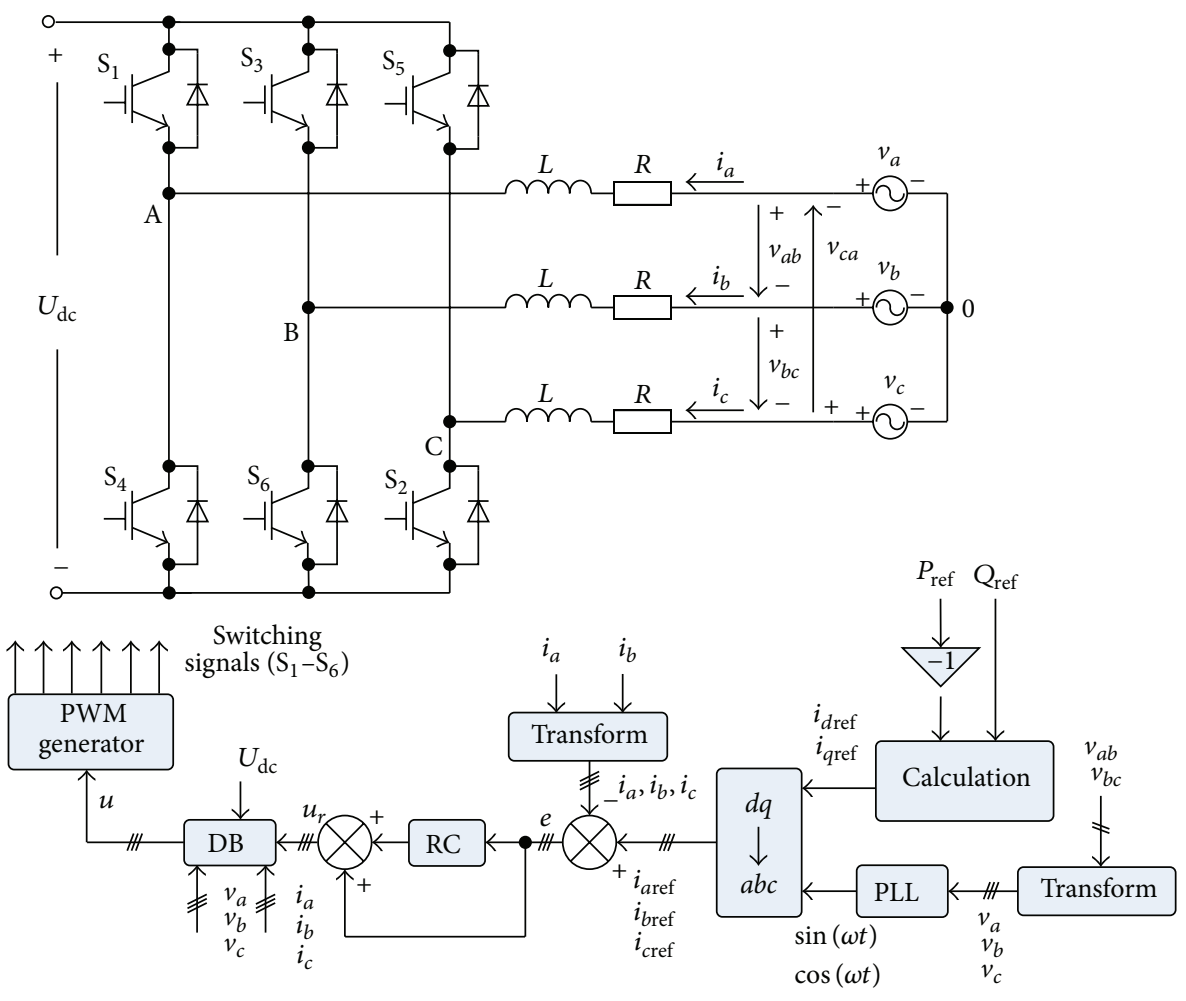

FIGURE 3: Repetitive controlled three-phase grid-connected PWM inverter.

the control gain of SHRC is $n / 2$ multiple of that of CRC. The error convergence rate of SHRC is also $n / 2$ times faster than that of CRC. Particularly, for the three-phase PWM inverters application, the error convergence rate of SHRC with $n=6$ and $m=1$ is three times (i.e., $n / 2=3$ ) faster than that of CRC. For the single-phase PWM inverters application, the error convergence rate of SHRC with $n=4$ and $m=1$ is two times (i.e., $n / 2=2$ ) faster than that of CRC. Therefore, SHRC can be used to enhance the error convergence rate for tracking/removing specific harmonics and thus improve the performance of three-phase/single-phase PWM inverter control system.

In the next two sections, SHRC will be used in threephase and single-phase grid-connected PWM inverter systems to verify the effectiveness of SHRC and to improve the control performance of both inverter systems.

\section{Case 1: Three-Phase Grid-Connected PWM Inverters}

4.1. Modeling. Figure 3 shows a three-phase grid-connected PWM inverter system, where $U_{\mathrm{dc}}$ is the dc-side voltage; $L$ and $R$ are inductance and resistor, respectively; $i_{a}, i_{b}$, and $i_{c}$ are the inductance currents; $v_{a}, v_{b}$, and $v_{c}$ are the $a, b$, and $c$ three-phase grid voltages. The control objective is to achieve high current tracking accuracy through forcing $i_{a}, i_{b}$, and $i_{c}$ to exactly track their reference $i_{\text {aref }}, i_{b \text { ref }}$, and $i_{c r e f}$, which are generated by using PQ control method and instantaneous power theory [26]. System parameter values are shown in Table 1.
TABLE 1: Parameters for three-phase grid-connected inverter system.

\begin{tabular}{lc}
\hline & $U_{\mathrm{dc}}=50 \mathrm{~V}$ \\
Inverter & $L=5 \mathrm{mH}$ \\
& $R=0.5 \Omega$ \\
& $v_{a b, b c, c a}=25 \mathrm{~V}(\mathrm{rms})$ \\
\hline \multirow{2}{*}{ Reference } & $P_{\text {ref }}=100 \mathrm{~W}$ \\
& $Q_{\text {ref }}=0$ \\
\hline Switching frequency & $f_{s}=6 \mathrm{kHz}$ \\
\hline
\end{tabular}

The mathematical model [18] can be described as

$$
\begin{aligned}
\left(\begin{array}{l}
\dot{i}_{a} \\
\dot{i}_{b} \\
\dot{i}_{c}
\end{array}\right)= & \left(\begin{array}{ccc}
-\frac{R}{L} & 0 & 0 \\
0 & -\frac{R}{L} & 0 \\
0 & 0 & -\frac{R}{L}
\end{array}\right)\left(\begin{array}{l}
i_{a} \\
i_{b} \\
i_{c}
\end{array}\right) \\
& +\left(\begin{array}{c}
\frac{1}{L}\left(v_{a}-v_{A}\right) \\
\frac{1}{L}\left(v_{b}-v_{B}\right) \\
\frac{1}{L}\left(v_{c}-v_{C}\right)
\end{array}\right)
\end{aligned}
$$


The data-sample format of (29) can be gotten:

$$
\begin{aligned}
& \left(\begin{array}{c}
i_{a}(k+1) \\
i_{b}(k+1) \\
i_{c}(k+1)
\end{array}\right) \\
& =\left(\begin{array}{ccc}
\frac{b_{1}-b_{2}}{b_{1}} & 0 & 0 \\
0 & \frac{b_{1}-b_{2}}{b_{1}} & 0 \\
0 & 0 & \frac{b_{1}-b_{2}}{b_{1}}
\end{array}\right)\left(\begin{array}{c}
i_{a}(k) \\
i_{b}(k) \\
i_{c}(k)
\end{array}\right) \\
& +\left(\begin{array}{c}
\frac{1}{b_{1}} v_{a}(k)-\frac{1}{b_{1}} \frac{U_{\mathrm{dc}}(k)}{2} u_{a}(k) \\
\frac{1}{b_{1}} v_{b}(k)-\frac{1}{b_{1}} \frac{U_{\mathrm{dc}}(k)}{2} u_{b}(k) \\
\frac{1}{b_{1}} v_{c}(k)-\frac{1}{b_{1}} \frac{U_{\mathrm{dc}}(k)}{2} u_{c}(k)
\end{array}\right)
\end{aligned}
$$

where $u_{a}(k), u_{b}(k)$, and $u_{c}(k)$ are the three-phase duty cycles, $b_{1}=L / T$, and $b_{2}=R$. Therefore, three independent single subsystems can be decomposed into

$$
\begin{aligned}
i_{j}(k+1)= & \frac{b_{1}-b_{2}}{b_{1}} i_{j}(k)+\frac{1}{b_{1}} v_{j}(k) \\
& -\frac{U_{\mathrm{dc}}(k)}{2} \frac{1}{b_{1}} u_{j}(k),
\end{aligned}
$$

where $j=a, b, c$.

A dead-beat (DB) current controller is chosen as follows:

$$
u_{j}(k)=\frac{2}{U_{\mathrm{dc}}(k)}\left[v_{j}(k)-b_{1} i_{\text {ref }}(k)+\left(b_{1}-b_{2}\right) i_{j}(k)\right],
$$

where $j=a, b, c$. Letting $i_{\text {jref }}(k)=i_{j}(k+1)$, the closed-loop transfer function without RC is $H(z)=z^{-1}$. Therefore, the control delay for DB current controller is only one step, that is, one sample period, in theory, which can achieve fast dynamic response.

Using the parameter values in Table 1, the mathematical model for the three-phase grid-connected inverter system in (31) can be rewritten into

$$
i_{j}(k+1)=0.9833 i_{j}(k)+0.0333 v_{j}(k)-0.8333 u_{j}(k)
$$

and the DB current controller in (32) can be rewritten into

$$
u_{j}(k)=0.04 v_{j}(k)-1.2 i_{\text {jref }}(k)+1.18 i_{j}(k),
$$

where $j=a, b, c$.

4.2. Experimental Results. Figure 4(a) shows the experimental result with single DB controller. It can be seen that current distortion is relatively serious; that is, the current waveform is not a smooth sine wave.
TABle 2: Parameters for single-phase grid-connected inverter system.

\begin{tabular}{lc}
\hline & $U_{\mathrm{dc}}=50 \mathrm{~V}$ \\
Inverter & $L=5 \mathrm{mH}$ \\
& $R=0.5 \Omega$ \\
& $v_{s}=25 \mathrm{~V}(\mathrm{rms})$ \\
\hline \multirow{2}{*}{ Reference } & $P_{\text {ref }}=50 \mathrm{~W}$ \\
& $Q_{\text {ref }}=0$ \\
\hline Switching frequency & $f_{s}=6 \mathrm{kHz}$ \\
\hline
\end{tabular}

Figures 4(b), 4(c), and 5 show the steady-state response and the current error convergence histories with CRC controller and the proposed SHRC controller being plugged into the DB controlled three-phase grid-connected inverter, respectively. For comparability, both $\mathrm{RC}$ gains are $k_{\mathrm{rc}}=$ 0.2 . Figures 4(b) and 4(c) show the current distortions can be greatly improved with both CRC and SHRC controllers. Moreover, it can be clearly seen from Figure 5 that the current error convergence times for CRC and SHRC are $0.32 \mathrm{~s}$ and $0.12 \mathrm{~s}$, respectively. Therefore, the current error convergence rate of the proposed SHRC controller is nearly three times faster than that of CRC controller (as indicated in Section 3.3).

Therefore, the effectiveness of SHRC for three-phase gridconnected PWM inverter system and its advantage compared with CRC are verified.

\section{Case 2: Single-Phase Grid-Connected PWM Inverters}

5.1. Modeling. Figure 6 shows a single-phase grid-connected PWM inverter system, where $U_{\mathrm{dc}}$ is the dc-side voltage; $L$ and $R$ are inductance and resistor, respectively; $i_{s}$ is the inductance current; and $v_{s}$ is the grid voltage. The control objective is to achieve high current tracking accuracy through forcing $i_{s}$ to exactly track its reference $i_{\text {sref }}$ which is generated by using PQ control method and instantaneous power theory [26]. System parameter values are shown in Table 2.

The mathematical model can be described as

$$
\dot{i}_{s}=-\frac{R}{L} i_{s}+\frac{1}{L}\left(v_{s}-v_{\text {in }}\right) \text {. }
$$

The data-sample format of (35) can be gotten:

$$
\begin{aligned}
i_{s}(k+1)= & \frac{b_{1}-b_{2}}{b_{1}} i_{s}(k)+\frac{1}{b_{1}} v_{s}(k) \\
& -\frac{1}{b_{1}} U_{\mathrm{dc}}(k) u_{s}(k),
\end{aligned}
$$

where $u_{s}(k)$ is the single-phase duty cycles, $b_{1}=L / T$, and $b_{2}=R$.

A DB current controller is chosen as follows:

$$
u_{s}(k)=\frac{2}{U_{\mathrm{dc}}(k)}\left[v_{s}(k)-b_{1} i_{\text {sref }}(k)+\left(b_{1}-b_{2}\right) i_{s}(k)\right] \text {. }
$$

Letting $i_{\text {jref }}(k)=i_{j}(k+1)$, the closed-loop transfer function without $\mathrm{RC}$ is $H(z)=z^{-1}$. Therefore, the control delay for DB 


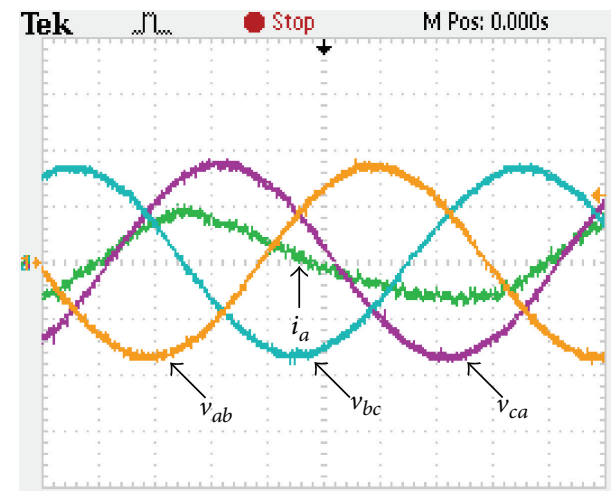

(a) DB

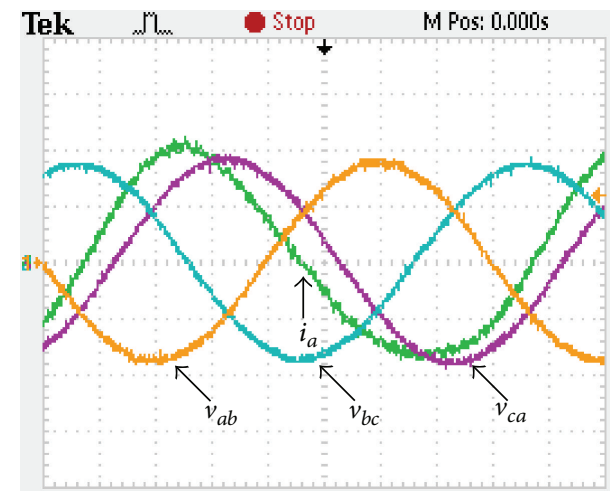

(b) $\mathrm{DB}+\mathrm{CRC}$

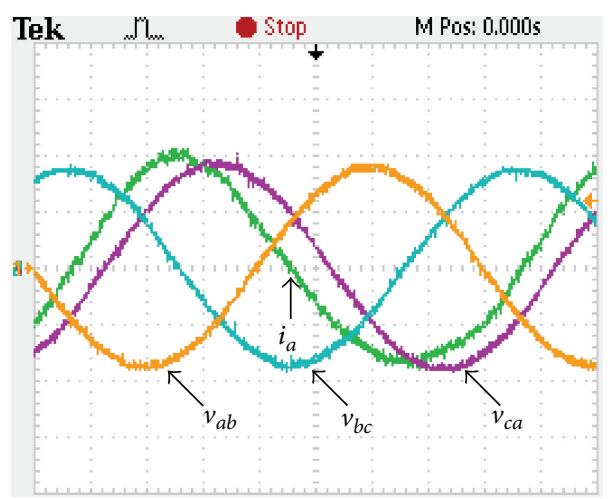

(c) $\mathrm{DB}+\mathrm{SHRC}$

FIGURE 4: Steady-state response for three-phase grid-connected inverter system ( $x$ label: $2.5 \mathrm{~ms} / \mathrm{div} ; y$ label: $20 \mathrm{~V} / \mathrm{div} \& 2 \mathrm{~A} / \mathrm{div}$ ).

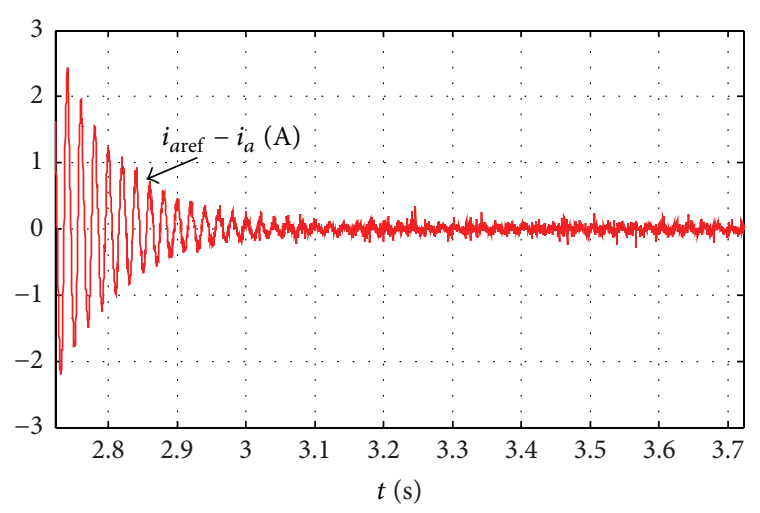

(a) CRC

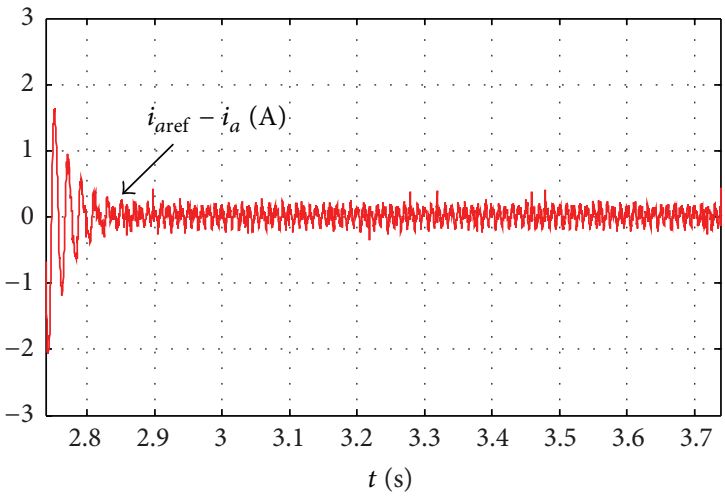

(b) SHRC

FIGURE 5: Current tracking error histories with two RCs plugged into for three-phase grid-connected inverter system.

current controller is only one step, that is, one sample period, in theory, which can achieve fast dynamic response.

Using the parameter values shown in Table 2, the mathematical model of the single-phase grid-connected inverter system in (36) can be rewritten into

$$
i_{s}(k+1)=0.9833 i_{s}(k)+0.0333 v_{s}(k)-0.4167 u_{s}(k)
$$

and the DB current controller can be used with the same coefficients in (34) for a convenient implementation purpose as follows:

$$
u_{s}(k)=0.04 v_{s}(k)-1.2 i_{\text {sref }}(k)+1.18 i_{s}(k) .
$$

5.2. Experimental Results. Figure 7(a) shows the experimental result with single DB controller. It can be seen that current distortion is relatively serious; that is, the current waveform is not a smooth sine wave.

Figures 7(b), 7(c), and 8 show the steady-state response and the current error convergence histories with CRC controller and the proposed SHRC controller being plugged into the DB controlled single-phase grid-connected inverter, respectively. For comparability, both $\mathrm{RC}$ gains are $k_{\mathrm{rc}}=$ 0.2 . Figures $7(\mathrm{~b})$ and 7 (c) show the current distortions for both CRC and SHRC controllers can be greatly improved. Moreover, it can be clearly seen from Figure 8 that the 


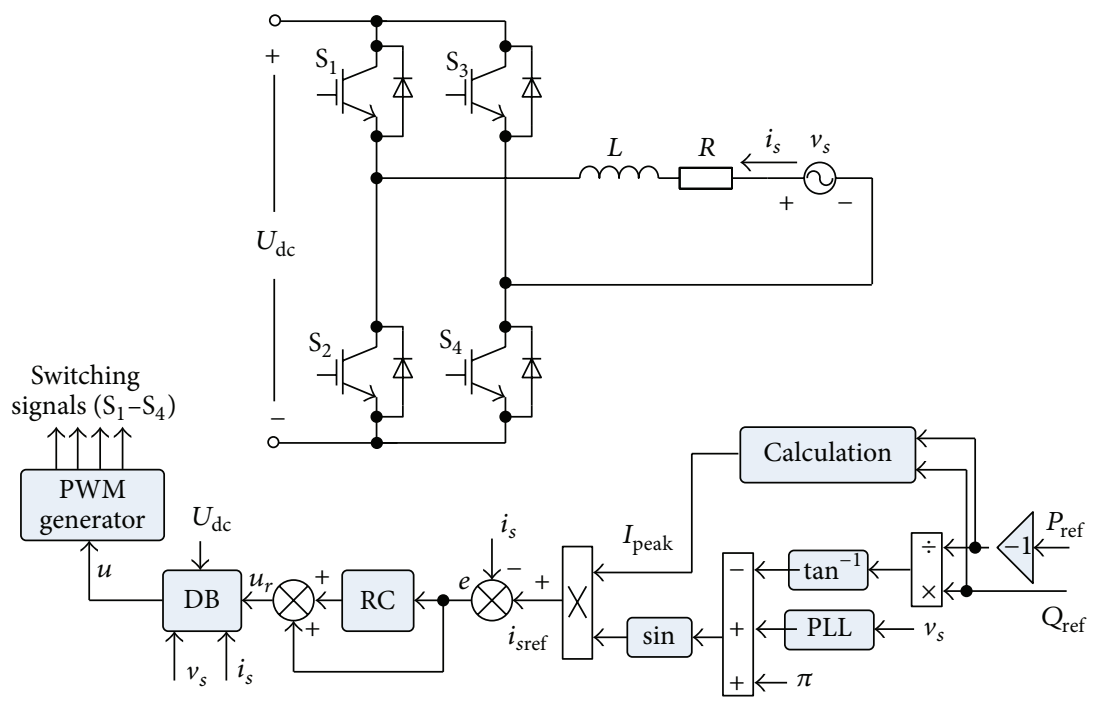

FIGURE 6: Repetitive controlled single-phase grid-connected PWM inverter.

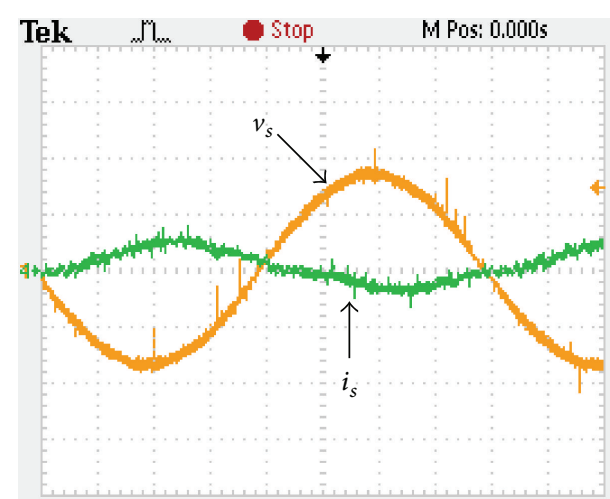

(a) $\mathrm{DB}$

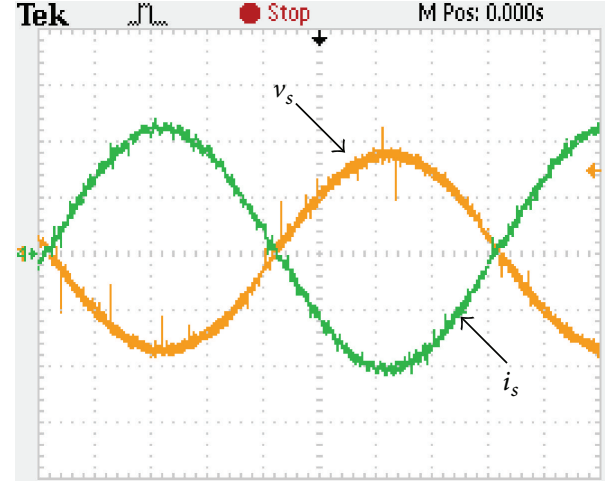

(b) $\mathrm{DB}+\mathrm{CRC}$

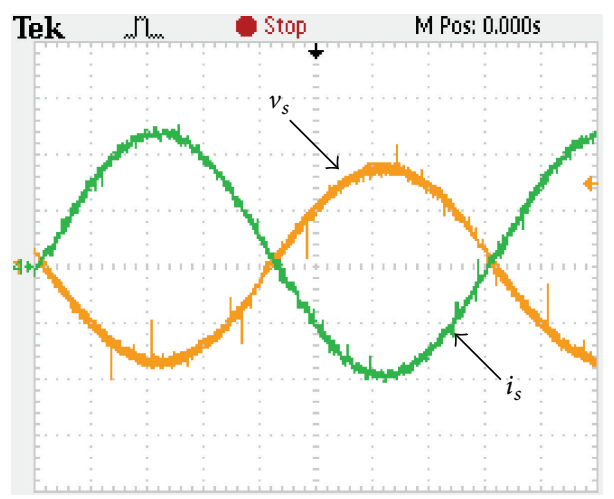

(c) $\mathrm{DB}+\mathrm{SHRC}$

FIgURE 7: Steady-state response for single-phase grid-connected inverter system ( $x$ label: $2.5 \mathrm{~ms} / \mathrm{div} ; y$ label: $20 \mathrm{~V} / \mathrm{div} \& 2 \mathrm{~A} / \mathrm{div}$ ).

current error convergence times for CRC and SHRC are $0.32 \mathrm{~s}$ and $0.16 \mathrm{~s}$, respectively. Therefore, the current error convergence rate of the proposed SHRC controller is nearly two times faster than that of CRC controller (as indicated in Section 3.3).

Therefore, the effectiveness of SHRC for single-phase PWM grid-connected inverter system and its advantage compared with CRC are verified.

\section{Conclusions}

This paper proposed an internal-model-principle-based (IMP-based) specific harmonics RC (SHRC). Using the general properties of IMP-based controller and the properties of exponential function, it can be concluded that SHRC can achieve zero-error tracking or perfect disturbance rejection of specific periodic signal only including these specific 


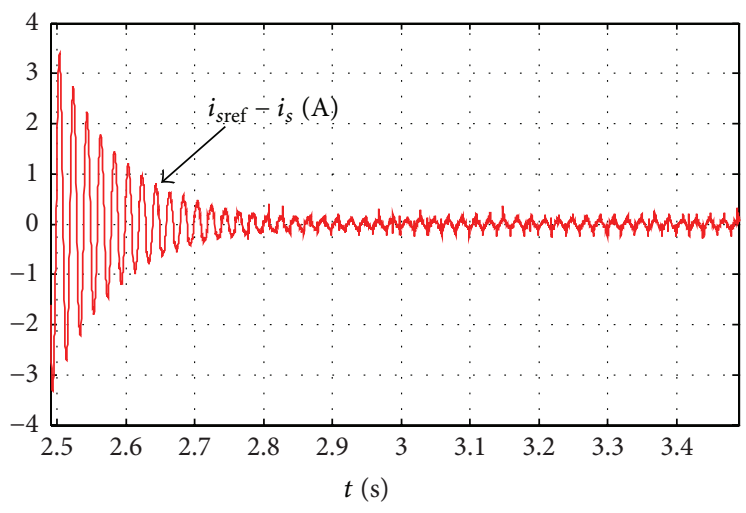

(a) CRC

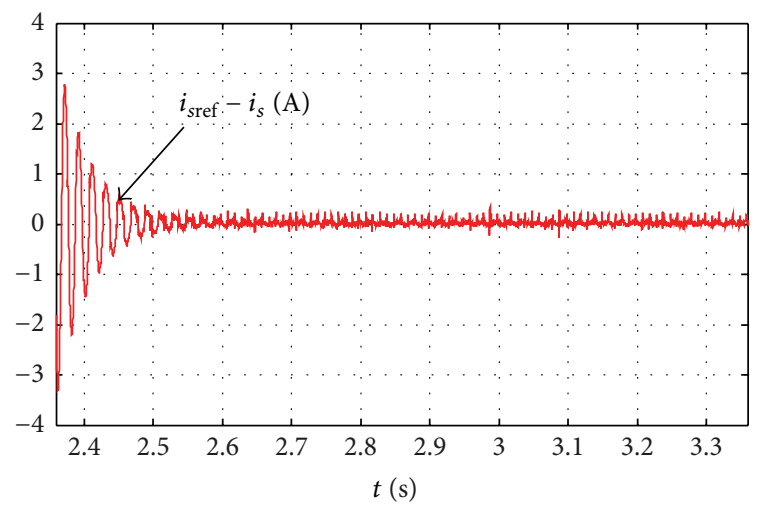

(b) SHRC

FIGURE 8: Current tracking error histories with two RCs plugged into for single-phase grid-connected inverter system.

$n k \pm m$ order harmonic components at $n / 2$ times faster error convergence rate compared with conventional RC (CRC).

Two application examples of SHRC controlled threephase/single-phase grid-connected PWM inverters have been given to show the effectives and promising advantages of SHRC. The experimental results show that, compared with CRC, SHRC offers three times (for three-phase inverter) or two times (for single-phase inverter) faster current error convergence rate and yields nearly the same current tracking accuracy. The proposed IMP-based SHRC provides a high performance control solution to grid-connected PWM inverters.

\section{Competing Interests}

The authors declare that there is no conflict of interests regarding the publication of this paper.

\section{Acknowledgments}

This work was supported in part by the Natural Science Foundation of China under Grant 51407084, by the Fundamental Research Funds for the Central Universities under Grant JUSRP11461, by the Jiangsu Province Science and Technology Support Project under Grant BE2013025, by the Natural Science Research key Project of Anhui Provincial Education Department under Grant KJ2014A026, by the National Natural Science Foundation of Anhui Provincial under Grant 1508085ME86, and by the Jiangsu Province Natural Science Foundation under Grant BK20130159.

\section{References}

[1] B. Francis, O. A. Sebakhy, and W. M. Wonham, "Synthesis of multivariable regulators: the internal model principle," Applied Mathematics and Optimization, vol. 1, no. 1, pp. 64-86, 1974.

[2] B. A. Francis and W. M. Wonham, "The internal model principle of control theory," Automatica, vol. 12, no. 5, pp. 457-465, 1976.

[3] S. Hara, Y. Yamamoto, T. Omata, and M. Nakano, "Repetitive control system: a new type servo system for periodic exogenous signals," IEEE Transactions on Automatic Control, vol. 33, no. 7, pp. 659-668, 1988.

[4] M. Tomizuka, "Zero phase error tracking algorithm for digital control," Journal of Dynamic Systems, Measurement, and Control, vol. 109, no. 1, pp. 65-68, 1987.

[5] M. Tomizuka, T.-C. Tsao, and K.-K. Chew, "Discrete-time domain analysis and synthesis of repetitive controllers," in Proceedings of the American Control Conference, pp. 860-866, Atlanta, Ga, USA, 1988.

[6] R. Grino and R. Costa-Castello, "Digital repetitive plug-in controller for odd-harmonic periodic references and disturbances," Automatica, vol. 41, no. 1, pp. 153-157, 2005.

[7] K. Zhou, K.-S. Low, D. Wang, F.-L. Luo, B. Zhang, and Y. Wang, "Zero-phase odd-harmonic repetitive controller for a singlephase PWM inverter," IEEE Transactions on Power Electronics, vol. 21, no. 1, pp. 193-201, 2006.

[8] R. Costa-Castelló, R. Grinó, and E. Fossas, "Odd-harmonic digital repetitive control of a single-phase current active filter," IEEE Transactions on Power Electronics, vol. 19, no. 4, pp. 1060 1068, 2004.

[9] B. Zhang, D. Wang, K. Zhou, and Y. Wang, "Linear phase lead compensation repetitive control of a CVCF PWM inverter," IEEE Transactions on Industrial Electronics, vol. 55, no. 4, pp. 1595-1602, 2008.

[10] G. Escobar, P. G. Hernandez-Briones, P. R. Martinez, M. Hernandez-Gomez, and R. E. Torres-Olguin, "A repetitivebased controller for the compensation of $6 \ell \pm 1$ harmonic components," IEEE Transactions on Industrial Electronics, vol. 55, no. 8, pp. 3150-3158, 2008.

[11] G. Escobar, P. R. Martínez, J. Leyva-Ramos, and P. Mattavelli, "A negative feedback repetitive control scheme for harmonic compensation," IEEE Transactions on Industrial Electronics, vol. 53, no. 4, pp. 1383-1386, 2006.

[12] K. Zhou, D. Wang, B. Zhang, Y. Wang, J. A. Ferreira, and S. W. de Haan, "Dual-mode structure digital repetitive control," Automatica, vol. 43, no. 3, pp. 546-554, 2007.

[13] K. K. Chew and M. Tomizuka, "Digital control of repetitive errors in disk drive systems," IEEE Control Systems Magazine, vol. 10, no. 1, pp. 16-20, 1990.

[14] C. Cosner, G. Anwar, and M. Tomizuka, "Plug in repetitive control for industrial robotic manipulators," in Proceedings of the IEEE International Conference on Robotics and Automation, vol. 3, pp. 1970-1975, Cincinnati, Ohio, USA, May 1990. 
[15] H. L. Broberg and R. G. Molyet, "Reduction of repetitive errors in tracking of periodic signals: theory and application of repetitive control," in Proceedings of the 1st IEEE Conference on Control Application, Dayton, Ohio, USA, 1992.

[16] K. Zhou, D. Wang, and K.-S. Low, "Periodic errors elimination in CVCF PWM DC/AC converter systems: repetitive control approach," IEE Proceedings-Control Theory and Applications, vol. 147, no. 6, pp. 694-700, 2000.

[17] K. Zhou and D. Wang, "Digital repetitive learning controller for three-phase CVCF PWM inverter," IEEE Transactions on Industrial Electronics, vol. 48, no. 4, pp. 820-830, 2001.

[18] K. Zhou and D. Wang, "Digital repetitive controlled three-phase PWM rectifier," IEEE Transactions on Power Electronics, vol. 18, no. 1, pp. 309-316, 2003.

[19] P. Mattavelli and F. P. Marafao, "Repetitive-based control for selective harmonic compensation in active power filters," IEEE Transactions on Industrial Electronics, vol. 51, no. 5, pp. 10181024, 2004.

[20] W. Lu, K. Zhou, D. Wang, and M. Cheng, "A general parallel structure repetitive control scheme for multiphase DC-AC PWM converters," IEEE Transactions on Power Electronics, vol. 28, no. 8, pp. 3980-3987, 2013.

[21] W. Lu, K. Zhou, D. Wang, and M. Cheng, "A generic digital $n k \pm m$-order harmonic repetitive control scheme for PWM converters," IEEE Transactions on Industrial Electronics, vol. 61, no. 3, pp. 1516-1527, 2014.

[22] W. Lu, K. Zhou, and D. Wang, "General parallel structure digital repetitive control," International Journal of Control, vol. 86, no. 1, pp. 70-83, 2013.

[23] G. C. Goodwin, S. F. Graebe, and M. E. Salgado, Control System Design, Prentice Hall, Upper Saddle River, NJ, USA, 2001.

[24] I. S. Gradshteyn and I. M. Ryzhik, Table of Integrals, Series, and Products, Academic Press, San Diego, Calif, USA, 7th edition, 2007.

[25] W. Lu, K. Zhou, and Y. Yang, "A general internal model principle based control scheme for CVCF PWM converters," in Proceedings of the 2nd IEEE International Symposium on Power Electronics for Distributed Generation Systems (PEDG '10), pp. 485-489, Hefei, China, June 2010.

[26] H. Akagi, H. E. Watanabe, and M. Aredes, Instantaneous Power Theory and Applications to Power Conditioning, Wiley, Hoboken, NJ, USA, 2007. 


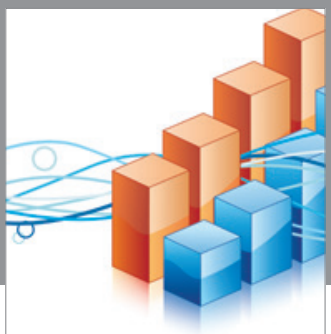

Advances in

Operations Research

vatem alat4

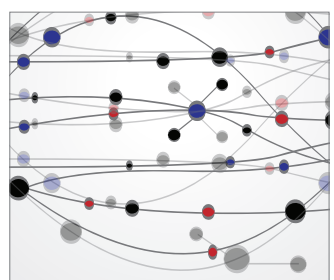

\section{The Scientific} World Journal
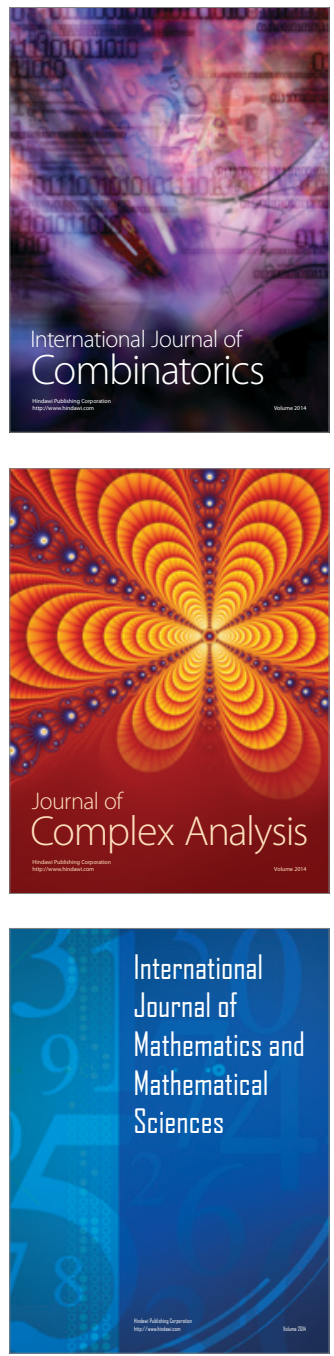
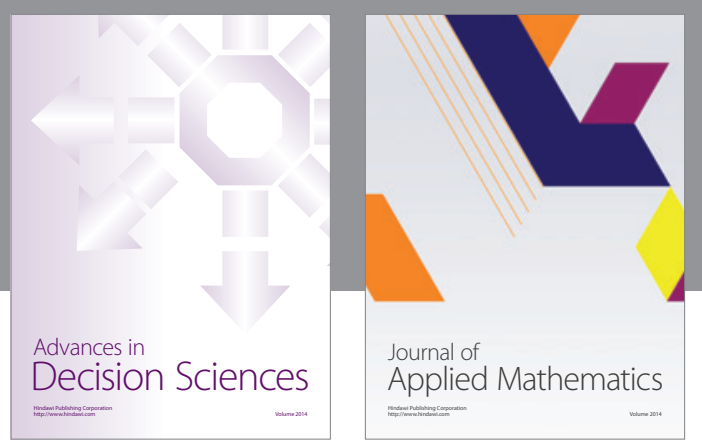

Algebra

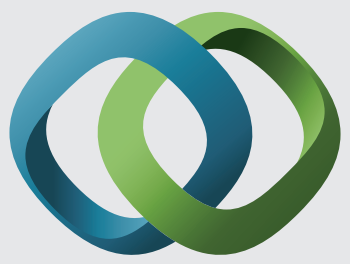

\section{Hindawi}

Submit your manuscripts at

http://www.hindawi.com
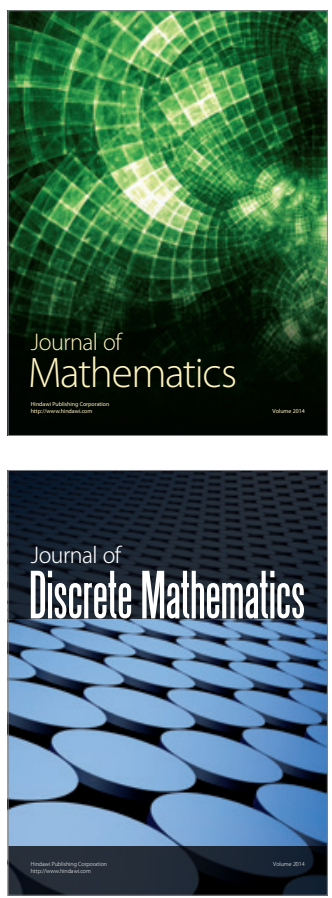

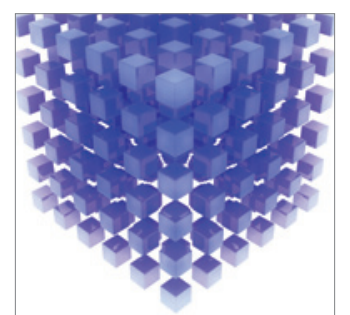

Mathematical Problems in Engineering
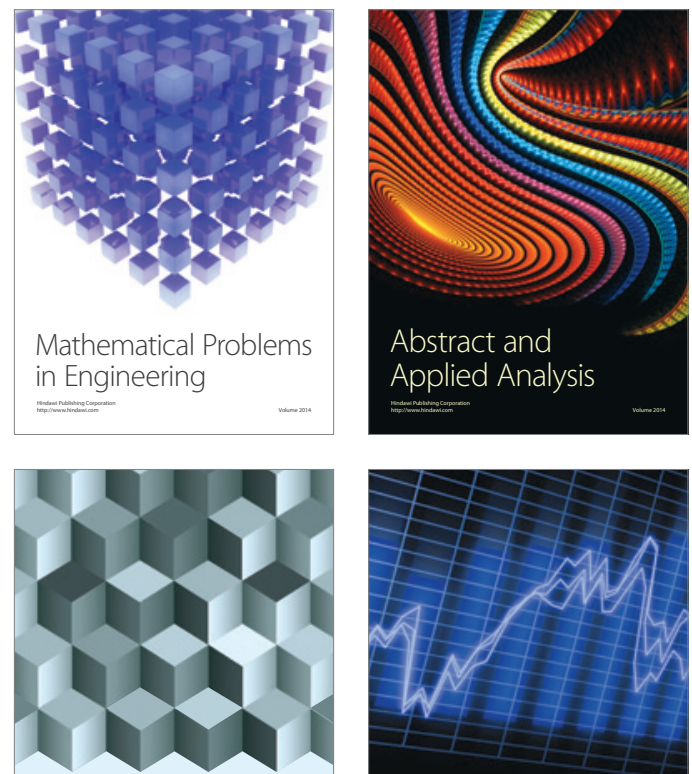

Journal of

Function Spaces

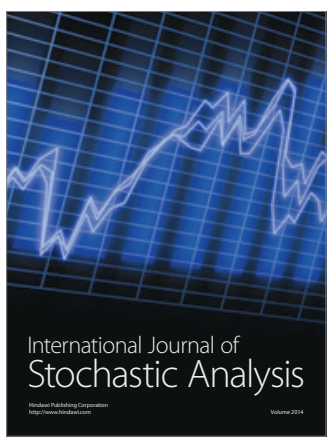

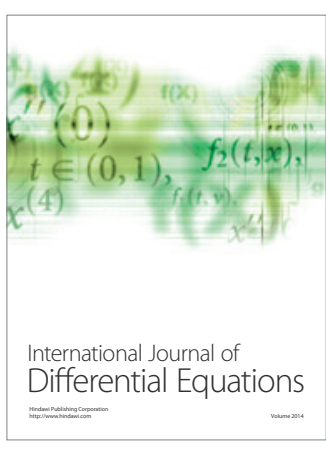
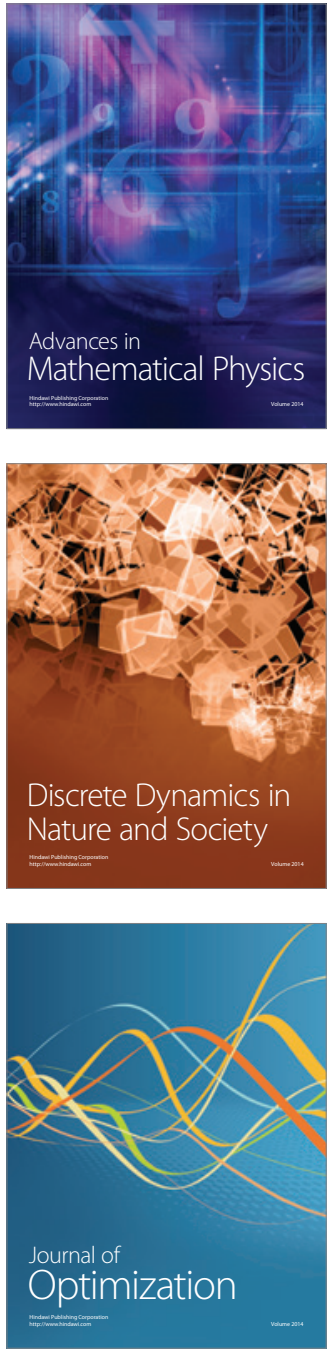